\title{
Uso y apropiación de las TIC: una exploración del acceso a los cibercafés y Kioskos Vive Digital en comunidades rurales*
}

\author{
Juan Camilo Paulhiac Pérez \\ Antonio José Ortega Hoyos ${ }^{* * *}$
}

Recibido: 25 de julio de 2018 - Aprobado: 28 de enero de 2019

El presente artículo abarca la relación entre las TIC y el desarrollo desde un enfoque cultural, tomando como caso de estudio los cibercafés y los Kioscos Vive Digital en Clemencia y María la Baja, dos localidades donde las TIC juegan un papel estratégico para el desarrollo rural. El estudio propone un diagnóstico inicial del estado de las TIC en ambas localidades y experimenta una metodología empírica en el estudio de los procesos de apropiación de las TIC, basada en la combinación de observaciones en campo con análisis de historiales de navegación en internet. El texto comenta acerca de cómo un mejor entendimiento de las subjetividades y de los usos recreativos de la tecnología digital puede mejorar la adquisición de competencias digitales en contextos donde las TIC juegan un papel clave en el desarrollo regional.

Palabras clave: cultura, entretenimiento, tecnología de la información, brecha digital, desarrollo humano, aprendizaje.

* Este artículo de investigación fue realizado en el marco del proyecto Laboratorios Vivos de Innovación y Cultura, implementado por la Universidad Jorge Tadeo Lozano, seccional Caribe en convenio con el Instituto de Cultura y Turismo de Bolívar (Icultur) 2015-2017. Financiado por el Fondo de Ciencia, Tecnología e Innovación del Sistema General de Regalías a través de la Gobernación de Bolívar. Artículo de investigación. Citar como Paulhiac, J. y Ortega, A. (2019). Uso y apropiación de las TIC: una exploración del acceso a los cibercafés y Kioskos Vive Digital en comunidades rurales. Análisis, 51(95), 289-318. DOI: https://doi.org/10.15332/21459169.4456

** Doctorado en Estética, Universidad Jorge Tadeo Lozano, Seccional Caribe (junio 2015-abril 2016). Investigador independiente (Colombia). Correo electrónico: juanpaulhiac@gmail.com. Orcid: http://orcid.org//0000-0002-8990-6436

*** Magíster en Gestión de la Innovación, Universidad Jorge Tadeo Lozano, seccional Caribe (junio 2015-octubre 2017). Docente de tiempo completo en Corporación Universitaria Rafael Núñez, Cartagena (Colombia). Correo electrónico: aortegah.18@gmail.com. Orcid: http://orcid.org//0000-0002-2985-7038 


\section{Use and appropriation of ICTs: An exploration of access to cyber cafés and Kioskos Vive Digital in rural communities}

Juan Camilo Paulhiac Pérez Antonio José Ortega Hoyos

\section{A bstract}

This article covers the relationship between ICTs and development from a cultural approach, taking as a case study the collective internet access points such as cyber cafés and Kioscos Vive Digital in Clemencia and Maria la Baja, two rural locations in northern Colombia, where ICTs play a key role in rural development. The study proposes an initial diagnosis of the state of ICTs in both locations and then experiments with an empirical methodology for studying the processes of appropriation of ICTs which combines fieldwork observations and web browser histories from collective access points. The text comments on how a better understanding of the subjective and recreational uses of technology may enhance the acquisition of digital literacy skills in contexts where ICTs play a key role in development processes.

Keywords: culture, entertainment, information technology, digital divide, human development, learning. 


\section{Usage et appropriation des TIC: Une étude sur l'accès aux cybercafés et kiosques Vive Digital dans des milieux ruraux}

Juan Camilo Paulhiac Pérez Antonio José Ortega Hoyos

\section{Résumé}

Cet article s'intéresse au rapport entre les TIC et le développement au travers d'une approche culturelle appliquée au cas des cybercafés et des kiosques Vida Digital à Clemencia et María la Baja, deux villages où les TIC jouent un rôle stratégique pour le développement rural. Cet étude propose un diagnostic sur l'état des TIC dans ces villages et met à l'épreuve une méthodologie empirique pour l'analyse des processus par lesquelles ces technologies sont appropriées. Cette méthodologie assemble des observations de terrain et des analyses de traces de navigation sur internet. Finalement, ce texte propose l'idée qu'entendement majeur des subjectivités et des usages ludiques de la technologie digitale peut améliorer l'acquisition de compétences digitales dans des contextes où les TIC sont censées contribuer au développement régional.

Mots clés: culture, loisir, technologie de l'information, écart digital, développement humain, apprentissage. 


\section{Introducción}

Los municipios de Clemencia y María la Baja están situados al norte del departamento de Bolívar, en la región Caribe colombiana (figura 1). Clemencia, municipio de unos 12.540 habitantes tiene una extensión de alrededor de $84 \mathrm{~km}^{2}$, de los cuales casi tres cuartas partes son área urbana. El municipio es atravesado por la vía principal que comunica Cartagena con Barranquilla y a nivel poblacional, ha vivido desde finales de los noventa un proceso de migración del campo hacia la cabecera municipal. En el 2015 el 78 \% de la población vivía en zona urbana (Paulhiac et al., 2016). A nivel educativo, en el 2013 la cobertura bruta en Clemencia fue de $109 \%$ (porcentaje de la totalidad de estudiantes matriculados en el sistema educativo) y la cobertura neta es de $87 \%$ (porcentaje de estudiantes matriculados en el sistema educativo; sin contar los que están en extraedad -por encima de la edad correspondiente para cada grado-) (Paulhiac et al., 2016). En cuanto a María la Baja, municipio de unos $547 \mathrm{~km}^{2}$, esta localidad cuenta con unos 48.079 habitantes (DANE, 2005). A diferencia de Clemencia, casi tres cuartos del territorio de María la Baja está ubicado en zona rural y el municipio está lejos de otras capitales departamentales. La población de María la Baja ha sido históricamente rural, en el 2005 con un 60 \% de la población viviendo fuera de la cabecera municipal. A nivel educativo, en el 2013 la cobertura bruta en María la Baja fue de $110 \%$ y su cobertura neta del $90 \%$.

Figura 1. Localización geográfica de los municipios de Clemencia y María la Baja

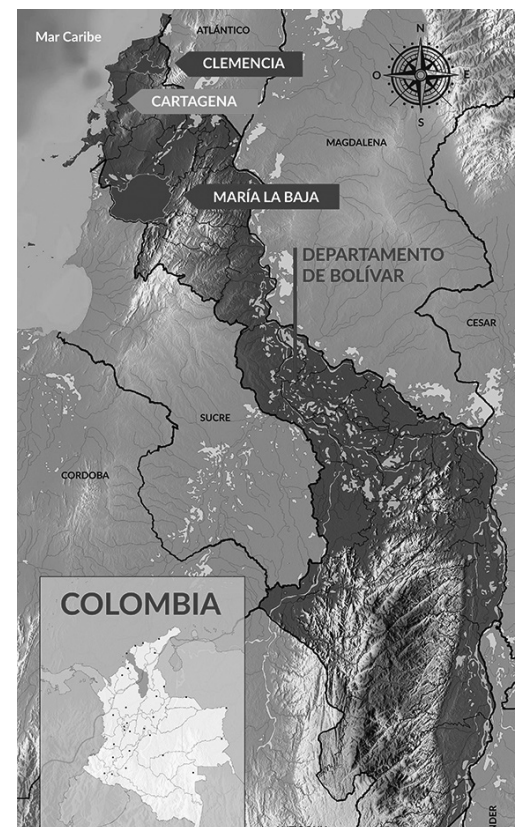

Fuente. Equipo de Laboratorios Vivos 
El presente estudio se sitúa en el contexto previo a la puesta en funcionamiento del proyecto "Laboratorios Vivos de Innovación y Cultura" en estos dos municipios, el cual tiene como objetivo "generar una estrategia para el uso y apropiación de la cultura como generadora de conocimiento e innovación social". La iniciativa, financiada por el Fondo General de Regalías, se inscribe dentro de una estrategia nacional para promover el desarrollo regional y se apoya en el proceso más amplio de expansión de las infraestructuras digitales, particularmente en zonas apartadas y de escasos recursos en Colombia.

Uno de los problemas sobre los cuales se fundamenta este proyecto ha sido la desarticulación entre las Tecnologías de la Información y las Comunicaciones (TIC) y el sector cultural, tal como lo demuestra la pobre infraestructura física y precariedad de infraestructura tecnológica de las casas de la cultura de Clemencia y María la Baja. Estas limitaciones, sin embargo, son sintomáticas de un problema más grande de cobertura de infraestructura digital en las zonas apartadas en Colombia. De acuerdo con información obtenida del Ministerio de Tecnologías de la Información y las Comunicaciones (2016), la penetración de internet en Clemencia y María la Baja no alcanza a llegar al 1 \% durante los dos últimos años (2014 y 2015). Mientras el municipio de Clemencia durante el primer trimestre de 2015 registraba una tasa de penetración de 0,9 $\%$, María la Baja solo alcanza el 0,4 \%.

Este acceso a internet se ha dado a través de los cibercafés y Kioscos Vive Digital (KVD) ubicados en los municipios. El primero es el nombre genérico con el cual se conocen habitualmente las iniciativas privadas que ofrecen acceso a computadores e internet como un negocio. En cuanto a los KVD, de acuerdo con el Ministerio de Tecnologías de la Información y las Comunicaciones (MinTIC, 2015), son puntos de acceso comunitario a internet, instalados en centros poblados (veredas y corregimientos) de más de 100 habitantes, donde sus usuarios pueden conectarse a internet y recibir capacitaciones gratuitas en uso y apropiación de las TIC. Los Kioscos Vive Digital hacen parte del Plan Vive Digital, que busca generar un ecosistema digital en todo el territorio colombiano, retomando los objetivos del programa Compartel, el cual abogaba por llevar internet a zonas remotas y de bajos recursos en el país.

Las características del contexto dan la oportunidad, cada vez más escasa, de observar los momentos iniciales de la llegada de la tecnología digital y la manera como esta permea el tejido social local. ¿Cómo comprenden las personas estas tecnologías, más allá del uso productivo que las instituciones prevén en el marco de los procesos de aprendizaje y apropiación de las TIC para el desarrollo? ¿Cómo se reflejan las subjetividades sociales en estos procesos de apropiación de la tecnología? Los municipios de Clemencia y María la Baja si bien son aquí casos de estudio, no son casos aislados. Al contrario, se asemejan a muchas otras poblaciones en Colombia y en general en América Latina,

1 Los Laboratorios Vivos son escenarios concebidos como ecosistemas, donde confluyen sinergias entre programas de formación y nuevas tecnologías para permitir la generación de procesos de innovación social, de emprendimiento y facilitar la sostenibilidad de la cultura en el Departamento de Bolívar. 
donde el recurso digital es incipiente y a la vez determinante en los planes de desarrollo regional. Entender mejor las dimensiones subjetivas inherentes al proceso de aprendizaje y apropiación de las TIC puede ayudar a entender mejor las características socioculturales del llamado "giro digital".

\section{Fundamentos teóricos}

\section{El campo de las TIC para el desarrollo}

En el marco de la llamada "fractura digital", entendida como la desigualdad en los niveles de acceso a las tecnologías de la información y de la comunicación (TIC) y en las capacidades de aprovechamiento del potencial de estas por falta de conocimientos (DiMaggio y Hargittai, 2004; Segev, 2010), es comúnmente aceptado que las TIC constituyen un eje estratégico para generar oportunidades de desarrollo en los contextos menos favorecidos.

Sobre esta premisa emerge desde comienzos de los años noventa en los medios académicos, las instancias gubernamentales, agencias diplomáticas y organizaciones no gubernamentales una discusión internacional y multidisciplinaria en torno al aporte de las TIC en el mejoramiento de las condiciones socioeconómicas en los países en desarrollo. En su análisis sobre este diálogo, Chrisanthi Avgerou (2010) ubica el campo de las TIC para el desarrollo (ICT4D) dentro del gran marco teórico del desarrollo humano propuesto por Amartya Sen (1985) y de los lineamientos planteados por los objetivos de desarrollo del milenio de la ONU (Avgerou, 2010).

Avgerou también clasifica un corpus representativo de la literatura en torno a dos grandes debates. El primero se centra en la cuestión de la innovación, articulando por un lado la idea de innovación como resultado de la transferencia e implantación de nuevas tecnologías y conocimientos y, por otro lado, la innovación como proceso endógeno de reorganización social frente a las nuevas tecnologías (se puede ubicar este segundo enfoque en el marco del concepto de innovación social que presenta Javier Echeverría, 2008). El segundo debate se centra en la cuestión del desarrollo, el cual se articula también entre dos perspectivas.

La primera es la de "transformación progresiva", según la cual las TIC en efecto permiten la transformación esperada de las condiciones sociales y las actividades humanas, este enfoque es característico de instituciones internacionales como el Banco Mundial (2012), el PNUD (Rocca y United Nations Development Programme, 2001) o la Comisión Económica para América Latina (CEPAL et al., 2013). 
La segunda es la de una "transformación disruptiva", característica por su posición crítica y de diversos cortes ideológicos frente a la idea de globalización y escéptica en cuanto a "la efectividad e intenciones de las políticas nacionales o internacionales sobre el desarrollo mediante las TIC" (Avgerou, 2010, p. 7).

Si bien estas discusiones hacen referencia a diversos aspectos de la relación entre TIC y desarrollo, Avgerou resalta las carencias teóricas que presenta la literatura en este campo de estudio, particularmente en lo referente a la cuestión cultural. Advierte que "las categorías de naciones, industrias u organizaciones formales", recurrentes en los textos, son insuficientes para entender la noción de contexto y que reducir la idea de "cultura local" a "suposiciones estereotipadas de comportamientos impide entender adecuadamente tanto el encuentro con nuevas tecnologías y las interacciones entre los múltiples actores involucrados en proyectos con las TIC, como sus consecuencias" (Avgerou, 2010, p. 11). ¿Cómo abordar entonces la dimensión cultural en la relación entre TIC y desarrollo más allá de estas categorías habituales?

\section{Consideraciones culturales acerca de las TIC para el desarrollo}

Más que una profundización teórica sobre los conceptos de cultura, TIC y desarrollo, nuestro propósito aquí es esbozar un marco funcional en la línea del campo de las TIC para el desarrollo, que ayude a entender cómo incide la subjetividad social en el proceso de adquisición de capacidades digitales (digital litteracy), tal como se evidencia empíricamente en este estudio.

Estas subjetividades pueden entenderse como imaginarios que se gestan y se nutren de la interacción con la tecnología y que evocan una dimensión cultural. En su descripción de la experiencia en el Media Lab "Cybermohalla", ubicado en uno de los suburbios de Delhi, Lawrence Liang reporta cómo a través de ejercicios de conversación y escritura los jóvenes participantes del laboratorio expresan sus diferentes expectativas en relación con su barrio, la tecnología, la cultura y la experiencia de vida en la ciudad. Liang plantea a raíz de estas experiencias de acceso a la TIC la construcción de "imaginarios digitales" vinculados a la vida comunitaria de estas personas, para al final preguntar, “¿cómo podemos empezar a observar la vida tecnológica de las personas más allá del desarrollismo, teniendo en cuenta la manera en que ello transforma las aspiraciones y las subjetividades?" (Liang, 2010, p. 66).

Esta perspectiva puede inscribirse dentro de lo que Arjun Appadurai ha llamado la "capacidad aspiracional" (Appadurai, 2004). Appadurai propone este concepto, apoyándose en la teoría del desarrollo humano de Amartya Sen, entre otras fuentes teóricas, para articular los campos del desarrollo y la cultura introduciendo en este último la noción de futuro. En efecto, Appadurai considera que hay una histórica contradicción intelectual entre los campos de cultura y desarrollo. Esto es porque la cultura, campo habitual de la antropología, ha 
tenido tendencia a ser referida durante más de un siglo haciendo alusión al pasado mediante términos como tradición, herencia, memoria, hábitos, etc., mientras que el desarrollo, campo de la economía, ha sido referido en alusión al futuro en términos de planificación, estrategia, objetivos, etc. (Appadurai, 2004, pp. 67-68).

Appadurai da crédito a los trabajos de Charles Taylor (1992) sobre el "reconocimiento" como fundamento ético del multiculturalismo, Albert Hirschman (1970) sobre la identificación colectiva, Amartya Sen sobre los valores sociales y el desarrollo (1984), las capacidades y el bienestar (1985) y la libertad (1999), Mary Douglas (1973-1982) sobre el contexto cultural de la anticipación al riesgo y James Fernández $(1965,1986)$ sobre la producción de consenso colectivo como fundamento epistemológico de la idea de "capacidad aspiracional".

Según sugiere Appadurai, hacer más explícita la idea de futuro en la cultura, es una manera de reducir la contradicción intelectual entre cultura y desarrollo, lo cual "podría tener implicaciones radicales para la pobreza y el desarrollo" (Appadurai, 2004). Es aquí donde aparecen las aspiraciones como una capacidad de las personas para proyectarse hacia el futuro. Esta capacidad aspiracional, argumenta Appadurai, es de tipo cultural, pues las aspiraciones "se forman siempre por la interacción y en el grueso de la vida social” (Appadurai, 2004) y, por ende, al igual que la memoria, hacen parte de los sistemas de pensamiento que conforman el ámbito sociocultural.

¿En qué medida las TIC, particularmente en contextos donde su acceso es reciente como en áreas rurales o apartadas de los países en desarrollo, representan un referente cultural de modernidad? La pregunta invita a pensar sobre los imaginarios y las aspiraciones que las TIC suscitan en las personas y la manera como desde la subjetividad se van generando procesos de apropiación social de la tecnología. Esto sugiere observar la relación entre cultura, TIC y desarrollo desde la sensibilidad que atañe la interacción con la tecnología, además del problema material del acceso a esta.

\section{Metodología}

El presente estudio combina un enfoque mixto, cualitativo y cuantitativo, basado en la aplicación de fuentes primarias (administradores, gestores y usuarios de espacios digitales) y fuentes secundarias (bibliografía relacionada). Las técnicas utilizadas en la recolección de información son principalmente la revisión documental, aplicación de encuesta directa, extracción de metadatos en registros de navegación en internet y contenidos en redes sociales digitales. Los datos primarios de este estudio fueron recolectados en dos fases sucesivas. Se agradece el apoyo de los auxiliares de investigación Kimberly Marín y Miller García del proyecto Laboratorios Vivos de Innovación y Cultura. 


\section{Fase 1: encuestas y observación en campo sobre usos y acceso a las TIC}

Como estrategia inicial para "entrar" en el terreno de los espacios públicos de acceso a las TIC, se aplicó una encuesta general de caracterización de dichos espacios enfocada a los administradores de los cibercafés y a los gestores de los Kioscos Vive Digital en los municipios de Clemencia y María la Baja, esta encuesta fue adaptada al objetivo del presente estudio según el modelo de encuesta propuesto en el estudio de Luis Fernando Barón y Ricardo Gómez (Barón y Gómez, 2010). Se requirió conocer, en primera instancia, los aspectos que en materia del uso de las TIC poseen los municipios a través de los cibercafés y los KVD. Los cibercafés fueron identificados progresivamente (muestreo por bola de nieve) consultando a miembros de la comunidad sobre la ubicación física de dichos espacios en su entorno. Para los KVD, se contó con un listado del Ministerio de Tecnologías de la Información y las Comunicaciones (Mintic, 2016) el cual permitió identificar un total de 3 kioscos funcionando en el municipio de Clemencia y 21 en María la Baja. En Clemencia fueron encuestados la totalidad de los espacios, mientras que en María la Baja se optó por efectuar un muestreo no probabilístico y se encuestó a 5 de los 21 espacios. En miras de un futuro estudio, un muestreo censal de los KVD en María la Baja permitirá una mayor granularidad en el análisis estadístico de registros de navegación (ver fase 2 de la metodología). Sin embargo, en cuanto a la encuesta de caracterización de los espacios digitales se considera suficiente el muestreo realizado, ya que la dotación tecnológica y el esquema de funcionamiento de los KVD es constante al depender directamente de una iniciativa gubernamental, por lo que encuestas adicionales resultarían redundantes.

Para la caracterización de los espacios se analizaron criterios como los servicios tecnológicos disponibles, los tipos de información más buscada por sus usuarios en la red, las actividades de los usuarios y operadores e información sociodemográfica general de usuarios. Adicionalmente, cada espacio tecnológico fue referenciado geográficamente mediante la aplicación Google Maps, con el fin de observar la dispersión espacial de los puntos de acceso y su relación con la ruralidad.

\section{Fase 2: levantamiento de datos digitales}

La segunda fase del estudio fue de carácter experimental. El propósito aquí fue explorar el alcance de los datos digitales (registros de historiales de navegación, contenidos en redes y sus respectivos metadatos) como fuente para elaborar una tipología de usos de las TIC en relación con los contextos culturales estudiados.

En primer lugar, se buscó extraer y analizar los registros de navegación de los equipos en los espacios tecnológicos. Esta práctica, que usualmente se aplica en la optimización de motores de búsqueda para estudiar y predecir las intenciones 
de los usuarios, se implementó aquí desde una perspectiva cultural para elaborar una tipología general de sitios visitados y para comprender el campo semántico de los términos clave utilizados en los motores de búsqueda. Estos registros permitieron entender aspectos generales sobre los espacios tecnológicos, tales como horarios y fechas de mayor y menor actividad y también detallar aspectos menos visibles como las categorías de las páginas consultadas, las cuales clasificamos según el índice WebPulse Site Review².

Así mismo, se establecieron las tendencias de búsqueda de información con los motores de Google.com y de Youtube.com. Se analizaron las búsquedas únicas por sesión, filtrando el registro por municipio, espacio tecnológico, número de equipo y finalmente código de tiempo, delimitando cada sesión a un lapso inferior a 30 minutos. En la mayoría de los casos este lapso coincidía con cambios temáticos en las búsquedas o páginas de inicio de sesión en portales de correo electrónico o redes sociales. Se hizo un análisis de frecuencia de frases y términos de búsqueda, por municipios y tipos de espacio tecnológico. Para este ejercicio se eliminaron los artículos y preposiciones para resaltar la dimensión semántica de los verbos y sustantivos empleados en las búsquedas. Posteriormente cada búsqueda fue clasificada manualmente tomando como referencia de indexación el índice dmoz.com. En miras de un estudio más amplio, sería conveniente integrar a la metodología las áreas de la ingeniería informática y la lingüística, con el fin de implementar técnicas de indexación automática.

\section{Resultados}

Los resultados que se presentan a continuación describen, en primera instancia, las principales características de los cibercafés y Kioscos Vive Digital ubicados en los municipios de Clemencia y María la Baja, Bolívar, a partir de su ubicación y tipología. La segunda parte hace referencia a los principales resultados de la encuesta aplicada, en torno a los usos que se dan en estos espacios. Por último, se hace referencia a los datos digitales levantados en los espacios tecnológicos.

\section{Espacios tecnológicos y territorio}

En cuanto al acceso a internet, al momento de realizar el presente estudio, los cibercafés y Kioscos Vive Digital eran los únicos espacios públicos de telecomunicación digital accesibles en ambos municipios. Un primer acercamiento georreferenciado de estos permitió apreciar su ubicación con respecto al tipo de espacio y a otros factores como la densidad poblacional y las principales vías de comunicación y de acceso. En ambos casos fue posible constatar la oferta 
de cibercafés dentro de las cabeceras municipales, al igual que la ausencia de Kioscos Vive Digital en estas cabeceras. Las figuras 1 y 2 evidencian también la relación de estos espacios con la ruralidad.

En el caso del municipio de Clemencia, en 2016 se identificaron cinco cibercafés para un poco más de 12.000 habitantes en la cabecera municipal, y tres Kioscos Vive Digital en los corregimientos de El Socorro, Piñique y Las Caras, para una población estimada de 2.000 habitantes (DANE, 2005). Las condiciones de acceso y el área del municipio de Clemencia y sus corregimientos permitieron visitar la totalidad de los establecimientos identificados. En total 5 cibercafés dentro del área urbana y 3 KVD ubicados en el área rural (figura 2).

Figura 2. Ubicación de cibercafés y Kioscos Vive Digital visitados en el municipio de Clemencia y corregimientos

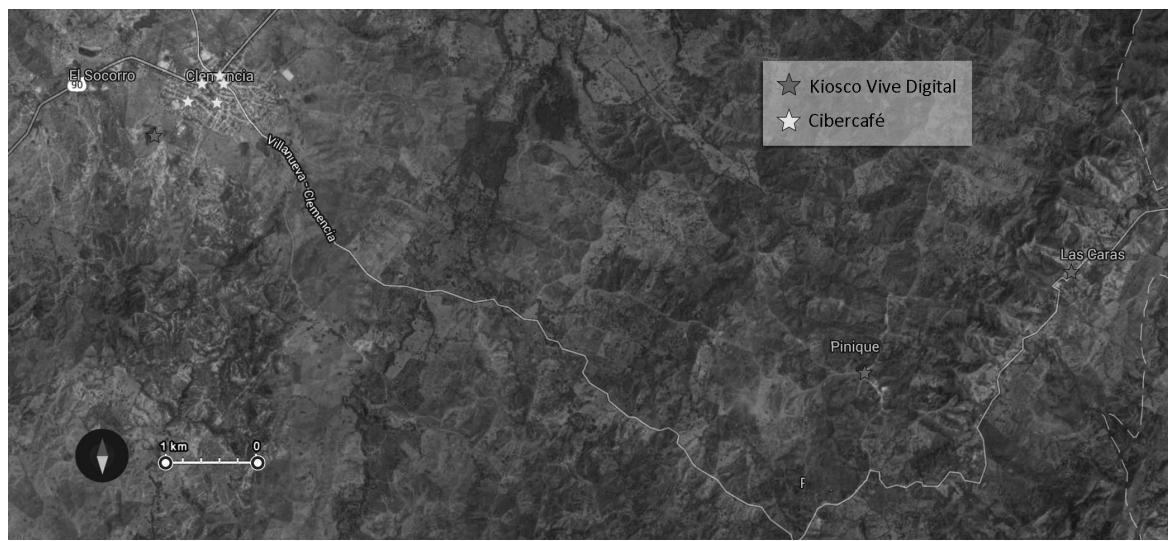

Fuente. Elaboración propia con base en Google Maps, 2016

En el caso de María la Baja, se identificaron cinco cibercafés en la cabecera municipal para unos 20.000 habitantes y 21 Kioscos Vive Digital en toda la zona rural del municipio, para una población de unos 28.000 habitantes. A diferencia de Clemencia, en María la Baja las distancias y dificultades de acceso obligaron a limitar las visitas. Si bien se tomaron la totalidad de los cibercafés, todos ubicados dentro del casco urbano, se realizó un muestreo de cinco KVD ubicados en la principal vía de acceso al municipio (figura 3).

En Clemencia existe también una mayor dispersión geográfica de los puntos de acceso de los corregimientos con respecto a la cabecera municipal. La totalidad de los espacios encuestados se encuentran dentro de un radio de 9 kilómetros, las vías de acceso a estas localidades no están pavimentadas y la movilidad es difícil. En María la Baja, existe mayor densidad de espacios en torno a la cabecera. Dentro de un radio de 4 kilómetros hay 11 espacios de conexión, incluyendo cibercafés y Kioscos Vive Digital. Las vías, aunque requieren mantenimiento, están pavimentadas y son de fácil acceso. 
Figura 3. Ubicación de cibercafés y Kioscos Vive Digital visitados en el municipio de María la Baja, corregimientos y veredas

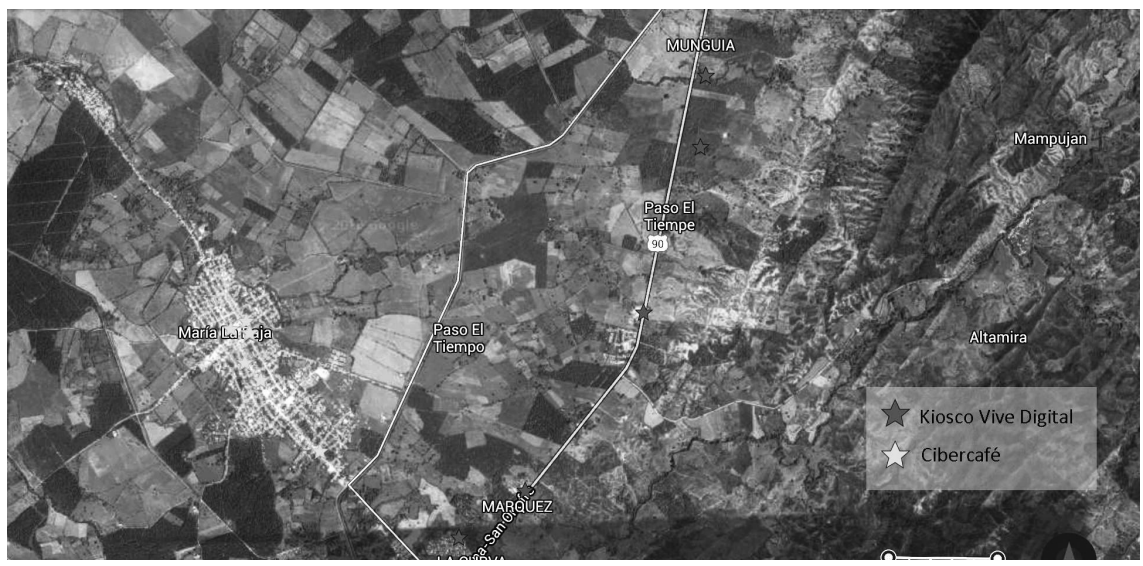

Fuente. Elaboración propia con base en Google Maps, 2016

\section{Tipología de espacios: cibercafés y Kioscos Vive Digital}

\section{Los cibercafés en los municipios (zona urbana)}

Los cibercafés han sido considerados como una alternativa de acceso a la red en contextos en desarrollo con poca cobertura universal, o de escasos recursos para adquisición de equipos personales. Estudios previos han identificado un rol importante de este tipo de espacios en el proceso de transición hacia la digitalización del acceso a la información y las comunicaciones. La literatura en este campo es amplia, pero para casos relevantes en América Latina, Lemos y Martini (2010) han estudiado la incidencia de este tipo de espacios (LAN-houses) en las industrias culturales brasileñas. Barón y Gómez (2010) hacen una comparación general de espacios de acceso público en Colombia.

En los casos de Clemencia y María la Baja, los cibercafés funcionan como en muchas zonas comparables ofreciendo servicio de acceso a internet, además de otros servicios de dactilografía, fotocopias o venta de variedades o accesorios, mediante lo cual este tipo de espacios se adapta a la evolución de la demanda local en materia de TIC. En ambos municipios las tarifas que se manejan en estos sitios son accesibles para el promedio de la población (desde 1000 pesos por hora), y los servicios se prestan en horarios de 12 horas normalmente, a partir de las 9 de la mañana hasta las 8 o 9 de la noche, durante toda la semana, e incluso los fines de semana.

Durante las visitas a estos municipios se evidenció una mejor organización en los cibercafés ubicados en el municipio de Clemencia (figura 4), los cuales tienen un mayor tiempo de funcionamiento con respecto a María la Baja. En Clemencia los primeros cibercafés empezaron a funcionar en el 2009, mientras 
que en María la Baja estos espacios aparecen desde el 2012. En Clemencia se destaca una mayor comodidad y privacidad para los usuarios con cubículos individuales en paneles, buena conectividad y equipos actualizados, así como presencia de equipos periféricos como cámara, audífonos y diferentes programas (software). Estas comodidades tienden a ser más escasas en los cibercafés de María la Baja, cuyo carácter parece más rústico (figuras 4 y 5).

Figura 4. Cibercafé Puntocom. Clemencia

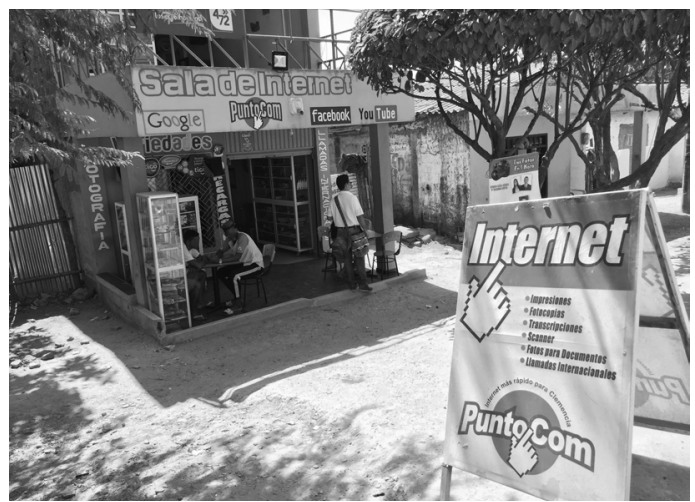

Fuente. Elaboración propia, 2016

Figura 5. Cibercafé Divina Stereo. María la Baja

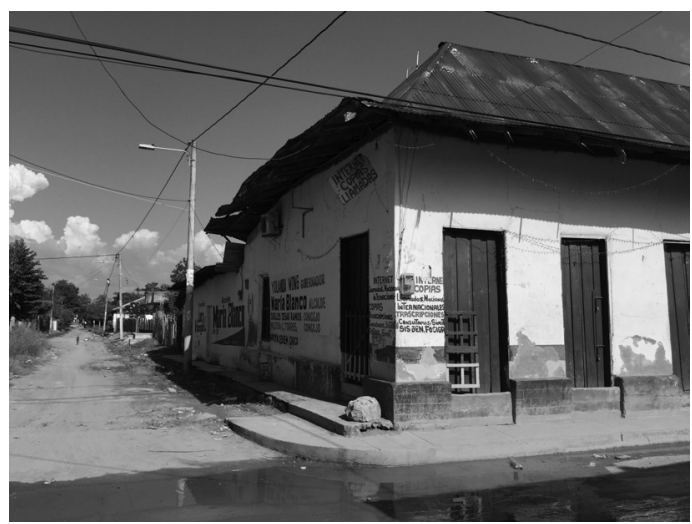

Fuente. Elaboración propia, 2016

Los administradores de estos sitios reportan que entre los servicios más vendidos están las fotocopias, escáner e impresiones. También es frecuente el servicio de llamadas telefónicas, el cual es "utilizado para atraer clientes" y generar ganancias adicionales. Entre otro tipo de servicios que brindan estos establecimientos, prevalece los trabajos de transcripciones o trabajos en computador, como producto de las tareas y actividades escolares que acuden a realizar los estudiantes. 
Desde el punto de vista de los usuarios, estos espacios han facilitado considerablemente el acceso a la información. Las personas entrevistadas reportan que es más fácil buscar información de tareas [pues] no necesariamente [en] un libro sale lo que uno necesita. Para otro usuario de los cibercafés, la ventaja de este tipo de espacio está en la facilidad de acceder a información sobre temas externos a la comunidad, la tecnología no estaba tan avanzada como ahora. [Se está] enterada de lo que pasa en el mundo [y se puede] crecer como persona (...) profesionalmente.

\section{Los Kioscos Vive Digital en los corregimientos y veredas (zona rural)}

Los Kioscos Vive Digital (KVD) son centros comunitarios de acceso a las TIC de iniciativa pública, que operan bajo las directrices del Ministerio de Tecnologías de la Información y las Comunicaciones (MinTIC), con el fin de implementar una política de acceso universal en las zonas rurales y apartadas del país mediante servicios de telefonía y conectividad en banda ancha. Los KVD están directamente ligados a los esfuerzos del gobierno para reducir la brecha digital y propiciar procesos de apropiación de la tecnología. Para ello, la dirección de conectividad del MinTIC incluye en la implementación de las políticas un fuerte componente de apropiación, a través del cual se busca capacitar a las personas en el uso productivo del internet, de manera que este sea utilizado como herramienta de desarrollo económico y social.

Figura 6. Kiosco Vive Digital del corregimiento de Montecristo (María la Baja)

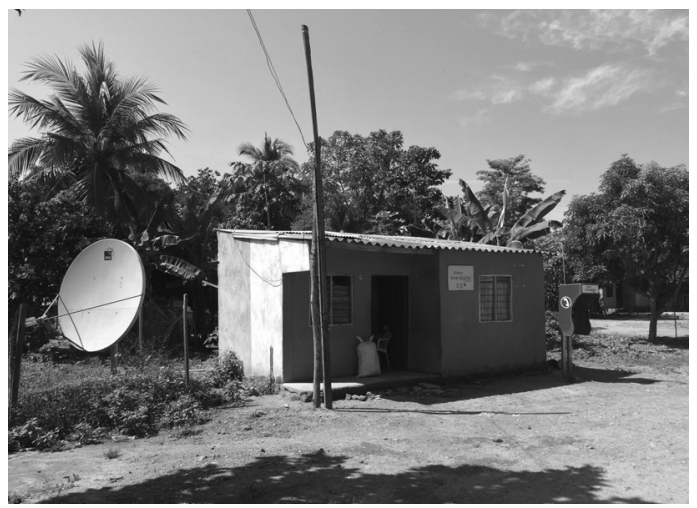

Fuente. Elaboración propia, 2016

Con base en los principales resultados obtenidos de la aplicación de encuestas a los gestores de los KVD, tenemos que existen varias similitudes con respecto a los cibercafés, pero también algunas diferencias. Por un lado, las tarifas en los KVD son inferiores a las de los cibercafés al estar subsidiadas por el gobierno nacional, y oscilan entre los 200 y 800 pesos por hora. Estos Kioscos prestan su servicio principalmente en sedes educativas, brindando conectividad a la comunidad escolarizada en la jornada escolar y en contrajornada atienden a la 
comunidad en general durante la semana, en diferentes horarios. Sin embargo, también funcionan de manera especial en algunas residencias de los habitantes de las localidades, como se evidencia en las figuras 6 y 7.

Figura 7. Kiosco Vive Digital del corregimiento de Piñique (Clemencia)

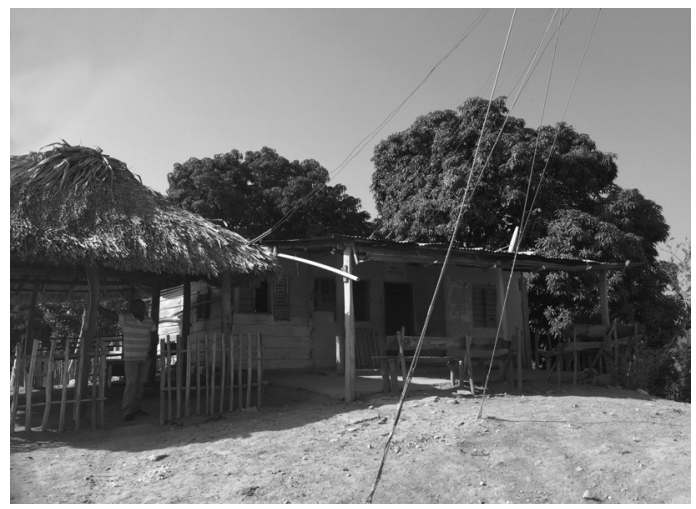

Fuente. Elaboración propia, 2016

Una característica primordial de este tipo de espacio es su función pedagógica en materia de TIC, la cual es garantizada por los gestores de cada espacio con base en una agenda nacional. La cual está organizada en módulos de formación de una media hora, donde se enseñan temas como "conocimiento general del computador", "aspectos generales de gobierno en línea", "correo electrónico" "redes sociales" o "entretenimiento en internet", entre otros. Estudios anteriores en Colombia (Barón y Gómez, 2014; Porras y Fernando, 2010), han caracterizado este tipo de espacios resaltando la participación mayoritaria de usuarios jóvenes y usos centrados en actividades personales, comunicación y redes sociales, y en particular actividades escolares. Esta última función se evidencia en las encuestas realizadas en campo. Algunos de los testimonios de usuarios, recogidos en campo, afirman que gracias a este tipo de espacio se puede encontrar fácilmente tareas de la universidad, (Sena); conseguir más información más rápido para ayudar en las tareas para los hijos (sic); o conseguir empleos y aspectos del estudio que [nos] ayudan a los jóvenes a actualizarnos.

\section{Análisis de usos de la tecnología a través de datos digitales}

El análisis de historiales de navegación permite tener una idea de las tendencias reales de la actividad en línea de los usuarios de los espacios tecnológicos estudiados. Los historiales son registrados automáticamente por aquellos navegadores instalados en los equipos que por defecto guardan la información de navegación una vez cerrada la sesión del computador. Los navegadores no conservan ningún tipo de registro único de usuario, por lo que los datos 
recopilados son anónimos. Cada registro está compuesto de cuatro campos: fecha, hora, dirección y dominio de cada página consultada.

El corpus bruto recopilado para este estudio fue de 18.053 entradas para tres cibercafés y tres Kioscos Vive Digital, en ambos municipios. Este corpus abarca un periodo de utilización entre agosto del 2015 y febrero del 2016, con intensidades de uso diferentes según los espacios y las fechas. Este corpus inicial fue ajustado a 11.906 entradas, manteniendo el mismo periodo inicial, pero omitiendo las secuencias de páginas consultadas dentro de redes sociales con un mismo dominio (por ejemplo Facebook.com), las cuales generaban una redundancia estadística. El corpus definitivo es resumido en la siguiente tabla.

Tabla 1. Muestreo ajustado de registros de navegación

\begin{tabular}{|l|l|c|}
\hline \multicolumn{1}{|r|}{ Espacio } & \multicolumn{1}{|c|}{ Municipio } & Registros \\
\hline Cibercafé 1 & Clemencia & 1561 \\
\hline Cibercafé 2 & Clemencia & 5510 \\
\hline Cibercafé 3 & María la Baja & 3237 \\
\hline KVD 1 & Clemencia & 148 \\
\hline KVD 2 & Clemencia & 513 \\
\hline KVD 3 & María la Baja & 937 \\
\hline TOTAL & & 11.906 \\
\hline
\end{tabular}

Fuente. Elaboración propia, 2016

En primera instancia, estos registros permiten corroborar algunos testimonios verbales realizados en las encuestas, a la vez que ayuda a afinar algunas observaciones. Por ejemplo, no se encontraron diferencias respecto a los horarios y días de acceso. Los KVD, según fue reportado por sus gestores en terreno, tienden a estar cerrados durante los fines de semana y suelen principalmente servir de apoyo para trabajos escolares. Mientras que los cibercafés tienen un uso más frecuente y se adaptan a una población adulta más abundante y por ende a usos más variados. Asimismo, el análisis de historiales muestra que los días de la semana con mayor actividad son los lunes para los cibercafés y los viernes para los KVD.

Los fines de semana la actividad en los cibercafés se mantiene constante, mientras que en los KVD prácticamente se detiene. En cuanto a los horarios, la actividad en los KVD es vespertina, ubicándose entre las 2 p. m. y las 5 p. m., con un pico de actividad hacia las 4 p. m. En el caso de los cibercafés, la actividad inicia desde las 8 a. m. y puede prolongarse hasta las 10 p. m., con un pico de actividad hacia las 7 p. m. 


\section{Tendencias de navegación}

Sin embargo, en algunos casos los registros de navegación permitieron ampliar significativamente las informaciones recogidas en campo mediante entrevistas, particularmente en cuanto a los usos reportados de internet. Entre las actividades TIC que con mayor frecuencia realizan los usuarios, de acuerdo con lo reportado por los administradores encuestados, están las actividades como chatear, navegar en web, acceder a las redes sociales e incluso ver videos en línea con los gustos musicales de los usuarios.

El análisis de historiales corrobora esta tendencia, pero también revela otras actividades según el tipo de espacio tecnológico. En el caso de los cibercafés, es de resaltar la visita frecuente a sitios para adultos, consulta general de páginas sobre tecnología, juegos, educación, sitios de empleo y descargas de contenidos. Para los KVD, se registra mayor énfasis en páginas de referencia (ayuda de tareas), juegos y contenidos para menores de edad, al igual que páginas de descargas de contenidos (figura 8 y 9).

Figura 8. Clasificación de páginas registradas en los historiales de navegación

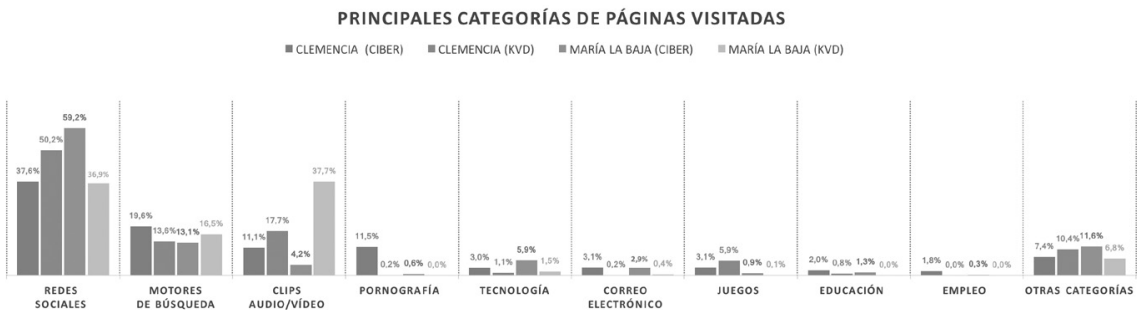

Fuente. Elaboración propia, según el índice de WebPulse Site Review, febrero de 2016

\section{Tendencias de búsquedas de información}

Otro aspecto por resaltar es la búsqueda de información a través de portales como Google. Estos son una herramienta fundamental para la clasificación y recuperación de informaciones en la web. De las 11.906 páginas registradas, 839 correspondieron a resultados de búsquedas en los motores de Google.com (67\%) y Youtube.com (33 \%). No se detectaron búsquedas en otros motores. De estas 839 búsquedas, aproximadamente 564 fueron búsquedas únicas por usuario o sesión (esta cifra es aproximada, ya que no se contó con un identificador específico de usuario). El análisis de las palabras clave y de las frases ingresadas permitió la clasificación de las búsquedas en temas y subtemas. La clasificación se hizo tomando en cuenta el sentido principal de la frase de búsqueda y no de cada palabra individual. La tabla 2 resume estos hallazgos en porcentaje de búsquedas por tema, para cada tipo de espacio y por municipio. 
Figura 9. Volúmenes de búsquedas en motores por municipio y tipo de espacio tecnológico

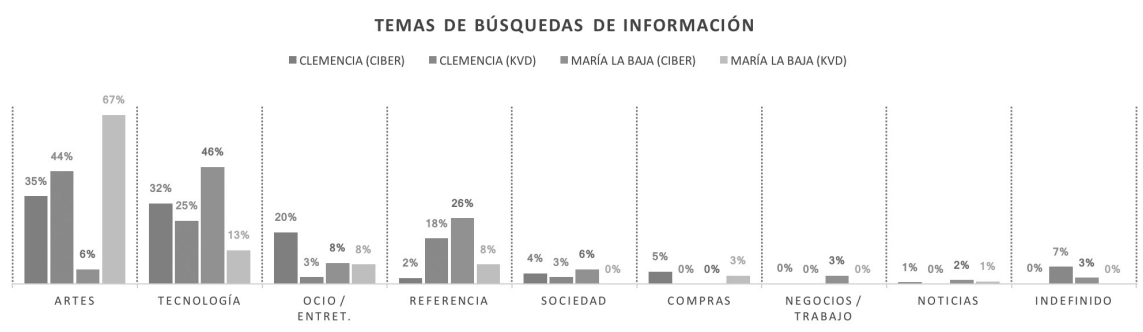

Fuente. Elaboración propia, según índice de Open Directory Project (dmoz.org)

Los registros evidencian una tendencia marcada general hacia búsquedas relacionadas a la música. Casi el $35 \%$ de todas las búsquedas realizadas conciernen a nombres de artistas, títulos de canciones o de álbumes y géneros musicales. En su mayoría se trata de artistas o géneros locales, como la champeta o el vallenato, aunque también son frecuentes los referentes extranjeros de hiphop o de reggaetón (tabla 2). Esta tendencia hacia la música es común a todos los espacios tecnológicos, excepto en los cibercafés de María la Baja, donde, aunque hay una buena diversidad de búsquedas de artistas musicales y de películas, existe un alto volumen de búsquedas de los nombres de servicios de redes sociales (Facebook) y de sus páginas de ayuda, así como los servicios de correo electrónico y los juegos en línea, por lo que en este caso las búsquedas sobre temas de tecnología son más frecuentes. Esta categoría es la segunda más frecuente en los demás espacios tecnológicos de los municipios. Allí se evidencia, particularmente, búsquedas de páginas de juegos en línea como "Juegos Friv". Otras búsquedas frecuentes en esta categoría son aplicaciones o software de edición o conversión de audio e imágenes, además de los ya mencionados dominios de redes sociales, páginas de ayuda de estos o motores de búsqueda.

La categoría de ocio y entretenimiento aparece globalmente como la tercera más solicitada, particularmente en los cibercafés. Las principales búsquedas en esta categoría son de contenidos pornográficos (solo desde los cibercafés), temas deportivos (fútbol) y reinados de belleza. En cuarto nivel de frecuencia están las búsquedas de información de referencia, las cuales tienen que ver con adquisición de nuevos conocimientos en general. Aquí son frecuentes las búsquedas de significados de palabras, conceptos o métodos que pueden a menudo ser asociados a las búsquedas de ayuda de tareas. Algunas frases típicas formuladas en esta categoría son, por ejemplo, "cuál es el continente más poblado" o "evolución de la vida política colombiana". Llama la atención en esta categoría algunas búsquedas sobre formas de mediación con otras personas, por ejemplo, frases "para confesar amor" o "para personas que fallecieron", así como información referencial sobre sexualidad (embarazos, etc.).

La categoría "sociedad" constituye menos del $4 \%$ de las búsquedas totales, y en casos como el de los KVD de María la Baja ninguna consulta se hizo en 
esta categoría. Las búsquedas más prominentes en esta categoría conciernen a instituciones educativas (por ejemplo, Sena o Icetex), temas de religión (Dios, letanías, etc.); mientras que los temas menos buscados fueron sobre vivienda, las propias localidades (búsquedas con las palabras Clemencia, María la Baja) y sobre servicios de gobierno. No se registraron búsquedas en temas de salud. La categoría "compras" no se refiere específicamente al hecho de comprar (por ejemplo, transacciones), ya que ninguna de las búsquedas en todo el corpus se refiere a esta acción en particular. Sin embargo, se conserva el nombre de la categoría del índice del directorio abierto de Google (dmoz.org), teniendo en cuenta que las subcategorías encontradas le corresponden. En efecto, se presentan búsquedas de vestuario y artículos de moda (vestidos de quinceañeras, vestidos de boda, zapatos, gorras y demás accesorios); temas de belleza corporal (mejores tatuajes, decoraciones de uñas), también se presentan, aunque en menor medida, búsquedas sobre motocicletas.

La categoría "negocios y trabajo" es de las menos solicitadas. En esta categoría se pueden resaltar algunas consultas técnicas (manejo de kardex) que bien podrían figurar bajo la categoría de "referencia", pero fueron clasificadas aquí por la especificidad temática que parece hacer alusión a una aplicación profesional de la información buscada. Otras búsquedas hacen mención del sistema de pagos (Datacrédito), y plataformas comerciales como Mercadolibre.com. Por último, la categoría "noticias" resume búsquedas de medios de comunicación, particularmente de prensa escrita (El Universal, Q'hubo), de canales de televisión (HTV) y de sucesos locales independientes de los medios (tiroteo en Clemencia).

Tabla 2. Categorías y subcategorías de las búsquedas de información por municipio y tipo de espacio

\begin{tabular}{|c|c|c|c|c|c|}
\hline \multirow{2}{*}{ Temas de búsqueda } & \multicolumn{2}{|c|}{ Clemencia } & \multicolumn{2}{|c|}{ María la Baja } & \multirow{2}{*}{ Total } \\
\hline & Ciber & KVD & Ciber & KVD & \\
\hline Artes & $34,6 \%$ & $44,4 \%$ & $5,9 \%$ & $66,7 \%$ & $34,9 \%$ \\
\hline Música & $31,8 \%$ & $41,7 \%$ & $2,5 \%$ & $46,7 \%$ & $29,3 \%$ \\
\hline Películas & $1,4 \%$ & $2,8 \%$ & $2,5 \%$ & $16,7 \%$ & $4,3 \%$ \\
\hline Series & $1,4 \%$ & $0,0 \%$ & $0,8 \%$ & $3,3 \%$ & $1,4 \%$ \\
\hline Tecnología & $31,8 \%$ & $25,0 \%$ & $46,2 \%$ & $13,3 \%$ & $31,0 \%$ \\
\hline Juegos & $13,1 \%$ & $1,4 \%$ & $5,9 \%$ & $1,1 \%$ & $8,2 \%$ \\
\hline Redes sociales & $5,3 \%$ & $5,6 \%$ & $16,0 \%$ & $0,0 \%$ & $6,7 \%$ \\
\hline Motores de búsqueda & $5,7 \%$ & $11,1 \%$ & $4,2 \%$ & $2,2 \%$ & $5,5 \%$ \\
\hline Aplicaciones & $2,5 \%$ & $5,6 \%$ & $5,9 \%$ & $2,2 \%$ & $3,5 \%$ \\
\hline
\end{tabular}




\begin{tabular}{|c|c|c|c|c|c|}
\hline \multirow{2}{*}{ Temas de búsqueda } & \multicolumn{2}{|c|}{ Clemencia } & \multicolumn{2}{|c|}{ María la Baja } & \multirow{2}{*}{ Total } \\
\hline & Ciber & KVD & Ciber & KVD & \\
\hline Correo & $1,8 \%$ & $1,4 \%$ & $9,2 \%$ & $2,2 \%$ & $3,4 \%$ \\
\hline Aprendizaje o tutoriales & $1,1 \%$ & $0,0 \%$ & $2,5 \%$ & $4,4 \%$ & $1,8 \%$ \\
\hline Telefonía & $1,4 \%$ & $0,0 \%$ & $0,8 \%$ & $0,0 \%$ & $0,9 \%$ \\
\hline Varios & $0,7 \%$ & $0,0 \%$ & $0,0 \%$ & $1,1 \%$ & $0,5 \%$ \\
\hline Descargas & $0,0 \%$ & $0,0 \%$ & $1,7 \%$ & $0,0 \%$ & $0,4 \%$ \\
\hline Series & $0,4 \%$ & $0,0 \%$ & $0,0 \%$ & $0,0 \%$ & $0,2 \%$ \\
\hline Ocio / entretenimiento & $20,5 \%$ & $2,8 \%$ & $8,4 \%$ & $7,8 \%$ & $13,7 \%$ \\
\hline Pornografía & $13,1 \%$ & $0,0 \%$ & $5,0 \%$ & $0,0 \%$ & $7,6 \%$ \\
\hline Deportes & $5,3 \%$ & $1,4 \%$ & $0,0 \%$ & $3,3 \%$ & $3,4 \%$ \\
\hline Varios & $1,8 \%$ & $0,0 \%$ & $0,8 \%$ & $4,4 \%$ & $1,8 \%$ \\
\hline Reinados & $0,0 \%$ & $1,4 \%$ & $2,5 \%$ & $0,0 \%$ & $0,7 \%$ \\
\hline Gastronomía & $0,4 \%$ & $0,0 \%$ & $0,0 \%$ & $0,0 \%$ & $0,2 \%$ \\
\hline Referencia & $2,5 \%$ & $18,1 \%$ & $26,1 \%$ & $7,8 \%$ & $10,3 \%$ \\
\hline Aprendizaje o tutoriales & $0,4 \%$ & $18,1 \%$ & $16,8 \%$ & $3,3 \%$ & $6,6 \%$ \\
\hline Ayuda personal & $0,4 \%$ & $0,0 \%$ & $6,7 \%$ & $2,2 \%$ & $2,0 \%$ \\
\hline Varios & $0,4 \%$ & $0,0 \%$ & $0,8 \%$ & $2,2 \%$ & $0,7 \%$ \\
\hline Sexualidad & $0,7 \%$ & $0,0 \%$ & $0,0 \%$ & $0,0 \%$ & $0,4 \%$ \\
\hline Relaciones personales & $0,0 \%$ & $0,0 \%$ & $1,7 \%$ & $0,0 \%$ & $0,4 \%$ \\
\hline Drogas & $0,4 \%$ & $0,0 \%$ & $0,0 \%$ & $0,0 \%$ & $0,2 \%$ \\
\hline Finanzas / economía & $0,4 \%$ & $0,0 \%$ & $0,0 \%$ & $0,0 \%$ & $0,2 \%$ \\
\hline Sociedad & $4,2 \%$ & $2,8 \%$ & $5,9 \%$ & $0,0 \%$ & $3,7 \%$ \\
\hline Educación & $1,4 \%$ & $0,0 \%$ & $3,4 \%$ & $0,0 \%$ & $1,4 \%$ \\
\hline Religión / espiritualidad & $0,7 \%$ & $2,8 \%$ & $0,8 \%$ & $0,0 \%$ & $0,9 \%$ \\
\hline Municipio & $0,7 \%$ & $0,0 \%$ & $0,8 \%$ & $0,0 \%$ & $0,5 \%$ \\
\hline Varios & $0,7 \%$ & $0,0 \%$ & $0,0 \%$ & $0,0 \%$ & $0,4 \%$ \\
\hline
\end{tabular}




\begin{tabular}{|c|c|c|c|c|c|}
\hline \multirow{2}{*}{ Temas de búsqueda } & \multicolumn{2}{|c|}{ Clemencia } & \multicolumn{2}{|c|}{ María la Baja } & \multirow{2}{*}{ Total } \\
\hline & Ciber & KVD & Ciber & KVD & \\
\hline Vivienda & $0,4 \%$ & $0,0 \%$ & $0,0 \%$ & $0,0 \%$ & $0,2 \%$ \\
\hline Gobierno & $0,0 \%$ & $0,0 \%$ & $0,8 \%$ & $0,0 \%$ & $0,2 \%$ \\
\hline Instituciones & $0,4 \%$ & $0,0 \%$ & $0,0 \%$ & $0,0 \%$ & $0,2 \%$ \\
\hline Compras & $4,9 \%$ & $0,0 \%$ & $0,0 \%$ & $3,3 \%$ & $3,0 \%$ \\
\hline Artículos de moda & $3,9 \%$ & $0,0 \%$ & $0,0 \%$ & $1,1 \%$ & $2,1 \%$ \\
\hline Belleza corporal & $0,7 \%$ & $0,0 \%$ & $0,0 \%$ & $2,2 \%$ & $0,7 \%$ \\
\hline Vehículos & $0,4 \%$ & $0,0 \%$ & $0,0 \%$ & $0,0 \%$ & $0,2 \%$ \\
\hline Indefinido & $0,4 \%$ & $6,9 \%$ & $2,5 \%$ & $0,0 \%$ & $1,6 \%$ \\
\hline Varios & $0,4 \%$ & $6,9 \%$ & $2,5 \%$ & $0,0 \%$ & $1,6 \%$ \\
\hline Negocios / trabajo & $0,4 \%$ & $0,0 \%$ & $3,4 \%$ & $0,0 \%$ & $0,9 \%$ \\
\hline Aprendizaje o tutoriales & $0,0 \%$ & $0,0 \%$ & $1,7 \%$ & $0,0 \%$ & $0,4 \%$ \\
\hline Comercio electrónico & $0,0 \%$ & $0,0 \%$ & $0,8 \%$ & $0,0 \%$ & $0,2 \%$ \\
\hline Finanzas / economía & $0,4 \%$ & $0,0 \%$ & $0,0 \%$ & $0,0 \%$ & $0,2 \%$ \\
\hline Búsqueda empleo & $0,0 \%$ & $0,0 \%$ & $0,8 \%$ & $0,0 \%$ & $0,2 \%$ \\
\hline Noticias & $0,7 \%$ & $0,0 \%$ & $1,7 \%$ & $1,1 \%$ & $0,9 \%$ \\
\hline Prensa & $0,4 \%$ & $0,0 \%$ & $1,7 \%$ & $0,0 \%$ & $0,5 \%$ \\
\hline Televisión & $0,0 \%$ & $0,0 \%$ & $0,0 \%$ & $1,1 \%$ & $0,2 \%$ \\
\hline Sucesos locales & $0,4 \%$ & $0,0 \%$ & $0,0 \%$ & $0,0 \%$ & $0,2 \%$ \\
\hline Total general & $100 \%$ & $100 \%$ & $100 \%$ & $100 \%$ & $100 \%$ \\
\hline Total de búsquedas únicas & 283 & 72 & 119 & 90 & 564 \\
\hline \multicolumn{6}{|c|}{ *El primer nivel de categoría fue adaptado del índice dmoz.org. } \\
\hline on las bús & ón & 6 & 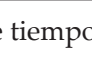 & & \\
\hline
\end{tabular}

Fuente. Elaboración propia, 2016

Adicionalmente a su clasificación temática, comparamos la frecuencia de los términos clave empleados en las búsquedas según el municipio, el motor de búsqueda y el tipo de espacio tecnológico. Se listaron las 10 primeras palabras 
para cada unidad de análisis. Para una mejor agregación de los datos, eliminamos de las frases los artículos y preposiciones. Sin embargo, en las tablas 3 y 4 fueron restituidas algunas de estas omisiones, preservando la formulación original hecha por los usuarios, para mayor claridad en la presentación.

Los resultados indican que la principal diferencia en los términos depende del motor de búsqueda empleado. En efecto, la gran mayoría de términos relacionados con contenidos artísticos o culturales (nombres de artistas y de picós, títulos de canciones y de álbumes) se hacen a través de YouTube, mientras que Google sirve para buscar información más variada. Esta tendencia se repite según el municipio observado, pero en las búsquedas en YouTube de Clemencia aparece una mayor presencia de búsquedas de picós, artistas y canciones de champeta, mientras que en María la Baja figuran primero nombres de artistas extranjeros que circulan en las redes de la industria mainstream.

Tabla 3. Palabras más buscadas en Clemencia

\begin{tabular}{|c|c|c|c|c|}
\hline \multirow{2}{*}{ N. ${ }^{\mathrm{o}}$} & \multicolumn{2}{|c|}{ Google } & \multicolumn{2}{|c|}{ YouTube } \\
\hline & Ciber & KVD & Ciber & KVD \\
\hline 1 & juegos friv & youtube & cancion & vol imperio \\
\hline 2 & $x x x$ & force & eclipse amor & rey de rocha vol \\
\hline 3 & facebook & facebook & $\begin{array}{l}\text { nicky jam } \mathrm{ft} \\
\text { kevin roldan }\end{array}$ & $\begin{array}{l}\text { mapale africano } \\
\text { tamboras }\end{array}$ \\
\hline 4 & youtube & convertidor & $x x x$ & el agua \\
\hline 5 & friv & ciudades & romeo santos & $\begin{array}{l}\text { twister ft mrl } \\
\text { dextany }\end{array}$ \\
\hline 6 & convertidor & medicion angulo & vol flow music & andy vol \\
\hline 7 & gmail & cuarteto & despues de amar & vol rs original \\
\hline 8 & youporn & reglas futbol & young $\mathrm{f}$ & $\begin{array}{l}\text { artista concierto } \\
\text { vol }\end{array}$ \\
\hline 9 & curn & verbos & j manny & $\begin{array}{l}\text { reina carnaval } \\
2016\end{array}$ \\
\hline 10 & vestidos 15 años & morfosintaxis & maluma & cancion \\
\hline
\end{tabular}

Fuente. Elaboración propia, 2016 
Tabla 4. Palabras más buscadas en María la Baja

\begin{tabular}{|c|l|l|c|l|}
\hline \multirow{2}{*}{ N.o } & \multicolumn{2}{|c|}{ Google } & \multicolumn{2}{c|}{ YouTube } \\
\cline { 2 - 5 } & \multicolumn{1}{|c|}{ Ciber } & \multicolumn{1}{|c|}{ KVD } & Ciber & \multicolumn{1}{c|}{ KVD } \\
\hline 1 & facebook & frases [de] vida & - & nik \\
\hline 2 & hotmail & kizoa & - & $\begin{array}{l}\text { maluma 2015 } \\
\text { diciembre }\end{array}$ \\
\hline 3 & youtube & youtube & - & signo rap \\
\hline 4 & outlook & mejores tatuajes & - & $\begin{array}{l}\text { peliculas completas } \\
\text { español }\end{array}$ \\
\hline 5 & friv & outlook & - & nicky jam \\
\hline 6 & frases [de] amor & $\begin{array}{l}\text { medir presion } \\
\text { persona }\end{array}$ & - & alia \\
\hline 7 & icetex & autos hermosos & - & otro lado \\
\hline 8 & q'hubo & peliculas & juegos & alkilados \\
\hline 9 & clima & clases narracion & - & tiros libres \\
\hline 10 & vol imperio & & & chris brown 2015 \\
\hline
\end{tabular}

Fuente. Elaboración propia, 2016

\section{Discusión}

Los resultados obtenidos en las diferentes fases del estudio invitan, por un lado, a considerar desde su complejidad, las interrelaciones de los contextos geográficos e institucionales con la "globalidad", desde los procesos de desarrollo vía las TIC en zonas rurales o "apartadas" en los municipios de Clemencia y María la Baja. Por otro lado, los resultados del comportamiento de navegación de los usuarios ofrecen una mirada sobre los usos de la tecnología digital y la información en el marco de procesos de aprendizaje y apropiación de las TIC, y del potencial impacto sociocultural de las tecnologías "globalizadas" en contextos "locales" de desarrollo.

\section{Lo subjetivo en la apropiación social de la tecnología}

Aunque los índices de penetración de las TIC en los municipios de Clemencia y María la Baja son bajos, es posible observar cómo los cibercafés y los KVD 
se complementan mutuamente para responder a la demanda local de acceso a las TIC. No obstante, más allá del acceso como primer indicador de desarrollo en contextos apartados, los usos de estas tecnologías evidencian un proceso de apropiación social fuertemente vinculado a la demanda y circulación de informaciones y contenidos de tipo cultural, tanto locales como internacionales.

Esto invita a observar el acceso a las TIC dentro de un habitus (Bourdieu, 1980) compuesto de mediaciones entre prácticas sociales subjetivas y estructuras institucionales, tanto políticas como educativas y tecnológicas. Cabe preguntar entonces, ¿en qué medida esta mediación es fuente de aspiraciones individuales, las cuales cobran sentido a nivel local, pero se construyen a través de prácticas como la búsqueda de informaciones en motores, o en la demanda de ciertos tipos de contenidos que circulan en esferas globales de comunicación?

En efecto, el solo acceso a un sistema de información "globalizado" no induce per se a una globalización cultural en términos de una estandarización de las aspiraciones individuales, o de los gustos culturales. Más bien podríamos plantear como hipótesis que en los contextos observados existe una propensión a la generación de sistemas de información locales más eficaces gracias a las plataformas virtuales, entre espacios tecnológicos en las cabeceras municipales y sus respectivos corregimientos. Estudios anteriores sugieren que espacios como los KVD han jugado un papel importante en la circulación de informaciones culturales dentro de los mercados culturales, como lo es el caso de la industria picotera y la música champeta en Cartagena de Indias (Paulhiac, 2014). Estos espacios, junto con herramientas como WhatsApp o Facebook, no solo sirven para trascender el espacio social físico, sino también para traer dentro de ese espacio informaciones, como agendas de eventos o influencias estéticas, que permiten dinamizarlo.

\section{El tiempo libre como estrategia de aprendizaje}

Los resultados del estudio indican que la gran mayoría del uso de la tecnología, tanto en cibercafés como en KVD, apunta hacia el consumo de contenidos de tipo cultural y de entretenimiento. La principal tendencia en la navegación es hacia la interacción vía redes sociales, la visualización de videos o películas, los juegos en línea y las búsquedas de información. En cuanto a estas últimas, los tipos de información solicitada vía los motores de Google y de YouTube corroboran esta tendencia.

Temas como la política, la economía, la salud o la educación, comúnmente considerados como más relevantes, o deseables, desde la perspectiva de las TIC para el desarrollo, figuran muy por debajo del promedio de navegación y de búsquedas de información. Esta tendencia se presenta inclusive en los KVD, espacios institucionales cuya función específica es precisamente pedagógica. 
Desde el punto de vista de las políticas de desarrollo vía las TIC, este patrón de comportamiento podría interpretarse como contraproducente. Es común priorizar contenidos de tipo "productivo" como la búsqueda de empleo, acceso a servicios de educación, o informaciones de noticias, etc., en la literatura sobre las TIC para el desarrollo. En su trabajo sobre la incidencia de Google en la profundización de la brecha digital en los usos de la información, Elad Segev (2010) cita varios trabajos (Bawden, 2001; Castells, 2005; Ciolek, 2003; Norris, 2008; Rogers, 2006; Webber y Johnston, 2000), en particular un estudio de Robert Putnam (2007) donde se demuestra una correlación positiva entre el nivel de compromiso cívico de una población dada y la cantidad de tiempo que esta dedica a ver noticias, versus un menor compromiso cívico y social con un mayor consumo de contenidos de entretenimiento como telenovelas o videojuegos (Segev, 2010, p. 84). Segev expone un círculo vicioso donde la posibilidad de elegir los contenidos mediáticos a través de los motores de búsqueda termina marginalizando a quienes, por falta de recursos intelectuales para hacer un uso efectivo de informaciones políticas y económicas, tendrán tendencia a permanecer en una esfera de información de ocio y entretenimiento.

Este argumento se basa sobre un índice de valor político y económico (EPV) distribuido en tres niveles de categorías: 1) las "categorías de nivel alto", son búsquedas de información sobre negocios, noticias, política, gobierno, organismos y leyes; 2) El nivel intermedio, son búsquedas sobre sociedad (aparte de las mencionadas en la categoría de nivel alto), información de referencia, ciencia, tecnología, región, hogar, salud y recreación;3) El nivel más bajo del índice son las búsquedas sobre arte, juegos y deporte (Segev 2010, p. 87). Si bien Segev sustenta de manera extensa su hipótesis del empoderamiento y el aumento del capital social a través de usos de información con mayor índice económico y político, este marco interpretativo deja varios interrogantes abiertos frente a los datos recogidos en el presente estudio.

El principal interrogante es sobre el proceso de aprendizaje y apropiación de la tecnología. En primer lugar, el aprendizaje técnico de las TIC implica una adquisición de conocimientos técnicos y herramientas intelectuales para hacer uso de las TIC. Esto obliga a relativizar el valor atribuible a los tipos de contenido mencionados por Segev en trabajos anteriores, en función del proceso de aprendizaje de los usuarios de las TIC, el cual es dinámico y progresivo. Esto aplica a los procesos de reciente apropiación en Clemencia y María la Baja, cuyos índices de penetración de las TIC, según se evidencia, son bastante bajos.

En estos contextos se requiere desarrollar competencias básicas clave antes de poder sacar mayor provecho de las TIC como herramienta de empoderamiento. Estas competencias, como por ejemplo la comprensión del funcionamiento de los motores de búsqueda y de sus mecanismos de personalización de datos, o la habilidad para desenvolverse estratégica e intencionalmente dentro de una red social virtual, pueden ser enseñadas, como de hecho lo son, mediante los programas pedagógicos de los KVD o a través de los gestores de espacios tecnológicos, pero fundamentalmente se aprenden de manera empírica. 
Esta familiarización con lo digital contribuye a generar el potencial necesario para el aprovechamiento de contenidos con alto valor económico y político, y puede por lo tanto ser visto como un momento clave en el proceso de empoderamiento mediante las TIC. En este sentido, sería prudente afirmar que los contenidos relacionados con el ocio y entretenimiento tienen un mayor valor con respecto a aquellos con alto índice político y económico.

Para los usuarios menos "alfabetizados" en materia de TIC y menos educados para hacer uso de los contenidos con un alto EPV, el consumo de contenidos de entretenimiento puede facilitar la adquisición de las mencionadas competencias. En efecto, actividades como buscar canciones o artistas favoritos o hacer uso intensivo de las redes sociales como Facebook, obligan a hacer un uso intensivo de los motores de búsqueda y a pensar el propio círculo social en términos de red hipertextual.

Otro interrogante tiene que ver con la manera en que el uso de la tecnología despierta y canaliza la expresión de aspiraciones individuales. Esto se evidencia, por ejemplo, en las búsquedas de información que parecen hacer referencia a cosas deseadas, gustos estéticos, tendencias, moda o criterios de belleza corporal. Algunos ejemplos de estas búsquedas son "imágenes de vestidos de 15 años", "uñas decoradas 2016", "champetas 2016", "gorras planas de miami heat", etc. Se pueden incluir aquí también aspiraciones más indirectas como las búsquedas de información referencial o de tutoriales que apuntan en ocasiones a aspiraciones educativas o profesionales, por ejemplo "sistema de medición de un ángulo", "cómo podría trabajarse el proceso de aprendizaje de la lectoescritura en preescolar y primero" o "manejo del kardex sistematizado". Por último, la progresiva ampliación del acceso a las TIC y el consecuente aumento de usuarios en un contexto social dado termina generando un habitus tecnológico al cual es normal aspirar para mantener una continuidad en las formas de relacionamiento con los demás.

Las tendencias de comportamiento que surgen del análisis de categorías temáticas sugieren que existe alguna forma de consenso social sobre las capacidades aspiracionales de los individuos en ese contexto (Appadurai, 2004). En este sentido, cabría preguntarse de qué manera la tecnología puede proveer, además de informaciones diversas para uso individual, un reflejo más general del "mapa aspiracional" de un contexto social dado (Appadurai, 2004).

Retomando la discusión de Appadurai expuesta líneas atrás, esto constituye un aspecto fundamental del campo cultural, y también puede ayudar a elaborar una hoja de ruta donde se puedan trazar vínculos más pertinentes entre las aspiraciones y la realización de estas. En este sentido, jerarquizar los usos de la tecnología parece menos pertinente para fines de desarrollo, que la comprensión de las aspiraciones reales y su relación con el marco de oportunidades y funcionamientos que ofrece el entorno social (Sen, 1985). 


\section{Conclusiones}

El acceso a las tecnologías digitales en contextos apartados o de escasos recursos, como los casos de los municipios de Clemencia y María la Baja, claramente ofrecen oportunidades para el desarrollo. Al respecto, la literatura hace énfasis en temas "productivos", directamente relacionados con indicadores socioeconómicos y de bienestar e inclusión social, como por ejemplo el acceso a servicios de educación, salud y gobierno, o la búsqueda de empleo y oportunidades de emprendimiento.

Sin embargo, aunque estos criterios parecen marcar la opinión que los usuarios tienen acerca de la utilidad de las tecnologías, los datos obtenidos a través del análisis cruzado de historiales de navegación y de observaciones in situ en este estudio apuntan hacia una marcada prevalencia de usos relacionados con el consumo de bienes culturales, particularmente musicales y actividades relacionadas con el ocio y el entretenimiento. Estos comportamientos son generalmente asociados por la literatura sobre TIC para el desarrollo como menos productivos, o al menos, con menor incidencia dentro de los procesos de desarrollo.

Frente a estos datos hemos buscado relativizar la jerarquía habitualmente establecida sobre los usos de las TIC para el desarrollo, tomando en consideración la capacidad del consumo de bienes culturales y de los usos relacionados con el ocio y el entretenimiento a fomentar el uso de las TIC y en particular a desarrollar habilidades clave para el aprovechamiento de la información, como lo son el aprendizaje del uso de los motores de búsqueda y la comprensión de la red como espacio social virtual. En consecuencia, argumentamos que el valor de los usos de las TIC en contextos de desarrollo, si bien depende del nivel de competencia y educación de los usuarios, también es relativo al momento del proceso de aprendizaje de estos.

Adicionalmente, desde una perspectiva cultural, encontramos necesario considerar los usos culturales de la tecnología a la luz de la capacidad aspiracional de las personas. Esto, por un lado, nutriría el diálogo sobre las TIC para el desarrollo, ampliando la perspectiva cultural más allá de los criterios de viabilidad de los proyectos en términos de productividad directa, pero, por otro lado, esto obligaría a pensar en las oportunidades reales que ofrece la estructura social, en términos de recursos económicos, educativos, alimentación, salud, etc., para alcanzar las aspiraciones que surgen a raíz de la apropiación de las TIC. Nuevos interrogantes de enfoque y metodología surgen: ¿Hasta dónde conviene considerar el ocio y entretenimiento como recurso de aprendizaje? ¿Cómo aprovechar los usos culturales de las TIC para dinamizar nuevas formas de participación ciudadana? ¿Cómo puede la tecnología y el big data ayudar a entender las tendencias aspiracionales en un contexto social, y qué implicaciones metodológicas y éticas conlleva esto? En suma, los resultados de este estudio dejan entrever la necesidad de investigaciones futuras y a mayor escala del tema en cuestión. 


\section{$\mathbf{R}_{\text {eferencias }}$}

Appadurai, A. (2004). The Capacity to Aspire: Culture and the Terms of Recognition. In V. Rao \& M. Walton (eds.), Culture and Public Action (pp. 59-84). Palo Alto, California: Recuperado de http://www.gsdrc.org/document-library/ the-capacity-to-aspire-culture-and-the-terms-of-recognition/

Avgerou, C. (2010). Discourses on ICT and Development. Information Technologies E International Development, 6(3), 1-18.

Banco Mundial. (2012). Information and Communication Technology (ICT) for greater development impact: World Bank Group strategy for ICT, (N. ${ }^{\circ}$ 73.236) (pp. 1-82). The World Bank. DOI: https://doi.org/10.1596/0-8213-5105-2

Barón, L. F. y Gómez, R. (2010). Acceso público a internet y cambio social: la experiencia en El Carmen de Bolívar, entre el silenciamiento y la esperanza. RECS CS, (6), 221-254. DOI: https://doi.org/10.18046/recs.i6.467

Barón, L. F. y Gómez, R. (2014). Más que teclas y pantallas. Acceso público a TIC en Colombia. Cali: Universidad Icesi. Recuperado de http://repository.icesi. edu.co/biblioteca digital/handle/10906/76575

Bawden, D. (2001). Information and digital literacies: A review of concepts. JD Journal of Documentation, 57(2), 218-259. DOI: https://doi.org/10.1108/ eum0000000007083

Bourdieu, P. (1980). Le sens pratique. Paris: Minuit.

Castells, M. (2005). Informationalism, networks, and the network society: A theoretical blueprinting. In The Network Society: A Cross-Cultural Perspective, (pp. 3-48). Cheltenham: Edward Elgar.

Ciolek, M. (2003). The Internet and its users: The physical dimensions of cyberpolitics in Eastern Asia. From the Book to the Internet: Communications Technologies, Human Motions, and Cultural Formations in Eastern Asia, University of Oregon: Research School of Pacific and Asian Studies, Australian National University.

Comisión Económica para América Latina (CEPAL), Deutsche Gesellschaft für Internationale Zusammenarbeit (GIZ), Bundesministerium für wirtschaftliche Zusammenarbeit und Entwicklung (BMZ), Unión Internacional de Telecomunicaciones (UIT), Ministerio de Medio Ambiente de Chile, \& EU. Commission of the European Communities (CEC). (2013). Las tecnologías de la información y de las comunicaciones (TIC) y el desarrollo sostenible en América Latina y el Caribe: experiencias e iniciativas de política. Memoria del seminario 
realizado en la CEPAL, Santiago, 22 y 23 de octubre de 2012. Recuperado de http://nbn-resolving.de/urn:nbn:de:101:1-2016042039543

Departamento Administrativo Nacional de Estadística - DANE. (2005). Censo general 2005: nivel nacional. Colombia: DANE.

DiMaggio, P. y Hargittai, E. (2004). Digital inequality: From unequal access to differentiated use. In K. Neckerman (ed.), Social inequality (pp. 355-400). New York: Russell Sage Foundation.

Echeverría, J. (2008). El Manual de Oslo y la innovación social. ARBOR Ciencia, Pensamiento y Cultura, 184(732), 609-618.

Lemos, R. y Martini, P. (2010). LAN Houses: A New Wave of Digital Inclusion in Brazil. Information Technologies \& International Development, 6(SE), 31-35.

MinTic. (2015). Ecosistema Digital. Recuperado de http://www.mintic.gov.co/ portal/vivedigital/612/w3-propertyvalue-634.html

Mintic. (2016). Puntos Vive Digital. Recuperado de https://www.mintic.gov.co/ portal/vivedigital/612/w3-propertyvalue-669.html

Norris, P. (2008). Digital divide: Civic engagement, information poverty, and the Internet worldwide. Cambridge: Cambridge Univ. Press. DOI: https://doi. org/10.1017/cbo9781139164887.001

Paulhiac, J. (2014). La scène musicale de la champeta face à Internet. Volume! La revue des musiques populaires, 10(2), 131-149. DOI: https://doi.org/10.4000/ volume.4073

Paulhiac, J., Ortega, A., Mendoza, L., Barraza, M., Ochoa, F., Santamaría, J. y Marín, K. (2016). Caracterización de las manifestaciones culturales en Clemencia y María la Baja (Bolívar). (Informe técnico, p. 107). Cartagena de Indias: Fundación Universidad de Bogotá Jorge Tadeo Lozano - seccional del Caribe.

Putnam, R. D. (2007). Bowling alone: The collapse and revival of American community. New York, NY: Simon \& Schuster.

Rocca, M. A. y United Nations Development Programme. (2001). Informe sobre desarrollo humano, Ecuador 2001: las tecnologías de información y comunicación para el desarrollo humano. Quito: Programa de las Naciones Unidas para el Desarrollo.

Rogers, R. (2006). Information politics on the Web. Cambridge, Mass: The MIT Press. 
Segev, E. (2010). Google and the Digital Divide: The Bias of Online Knowledge. Chandos Pub.

Sen, A. (1985). Commodities and capabilities. Amsterdam, New York, NorthHolland: Sole distributors for the USA and Canada, Elsevier Science Pub. Co.

Symantec. (n.d.). Symantec Sitereview. Retrieved from May 7, 2019, https://sitereview.bluecoat.com/\#/

Webber, S. y Johnston, B. (2000). Conceptions of information literacy: New perspectives and implications. Journal of Information Science, 26(6), 381-397. DOI: https://doi.org/10.1177/0165551004233401 